\title{
Silica polygonal micropillar resonators: Fano line shapes tuning by using a Mach-Zehnder interferometer
}

\author{
Kam Yan Hon and Andrew W. Poon \\ Department of Electrical and Electronic Engineering, The Hong Kong University of Science and \\ Technology, Clear Water Bay, Hong Kong SAR, China \\ Phone: 852-2358-7905, Fax: 852-2358-1485, email: eeawpoon@ust.hk
}

\begin{abstract}
We experimentally demonstrate Fano resonance line shapes tuning by using a Mach-Zehnder interferometer (MZI). We employ a silica $125-\mu \mathrm{m}$-size hexagonal micropillar resonator with prism coupling in one arm of the interferometer, and a phase shifter together with a variable attenuator in the other arm. Our initial experiments reveal that the resonance line shapes observed at the interferometer output are characteristically asymmetric as Fano resonances. By using the phase shifter, we controllably tune the asymmetric line shapes from near-symmetric dip to near-symmetric peak and back to near-symmetric dip. We discuss potential applications of our MZI-based microresonator resonance line shapes tuning for bio-chemical sensing.
\end{abstract}

Keywords: Fano resonances, resonance line shapes, micropillar resonators, Mach-Zehnder interferometer

\section{INTRODUCTION}

Fano resonances ${ }^{1}$ are ubiquitous in quantum and classical resonance systems. Fano resonances occur when a resonance pathway interferes with a coherent background pathway. The resultant resonance line shapes are characteristically asymmetric, often with a signature "dip" adjacent to the resonance, yet also exist in the form of a symmetric inverted Lorentzian. In optical systems, Fano resonances have long been recognized in grating diffraction and dielectric particles elastic scattering phenomena. Recently, optical Fano resonances have also been reported in various optical microcavities including integrated waveguide-coupled microcavities ${ }^{2}$, prism-coupled square micropillar resonators ${ }^{3,4}$, multimode tapered fiber coupled microspheres ${ }^{5}$, and Mach-Zehnder interferometer (MZI) coupled microcavities. ${ }^{6}$ It has been suggested that optical Fano resonances have niche applications in resonance line shape sensitive bio-sensing ${ }^{7}$, optical channel switching and filtering ${ }^{2-5}$. Previously ${ }^{3,4}$, we reported experimental observations and modeling of Fano resonances in prism-coupled square micropillar resonators. We analyzed the observed singlemode asymmetric resonance line shapes in terms of interference between a single resonance mode and a coherent background ${ }^{3}$, and the observed multimode asymmetric resonance line shapes in terms of interference between a high-Q resonance mode and a detuned low-Q resonance mode. ${ }^{4}$

Here we report an experimental demonstration of Fano resonance line shapes tuning by using an MZI, as shown in Fig.1. We employ a prism-coupled silica hexagonal micropillar resonator to generate singlemode resonances in one arm of the interferometer, and a phase shifter together with a variable attenuator in the other arm. Our initial experiments show that the interferometer output exhibits Fano resonances and the line shapes can be controllably tuned from a near-symmetric dip to a near-symmetric peak.

The paper is organized as follows: Section 2 reviews concept and modeling of optical Fano resonances. Section 3 presents principle and experiments of MZI-based Fano line shapes tuning. Section 4 discusses potential applications of controlled Fano line shapes tuning technique in (biochemical) sensing. Section 5 concludes the present work.

Laser Beam Control and Applications, edited by Alexis V. Kudryashov, Alan H. Paxton, Vladimir S. Ilchenko, Adolf Giesen, Detlef Nickel, Steven J. Davis, Michael C. Heaven, J. Thomas Schriempf, Proc. of SPIE Vol. 6101, 61010V, (2006) · 0277-786X/06/\$15 · doi: 10.1117/12.669567 


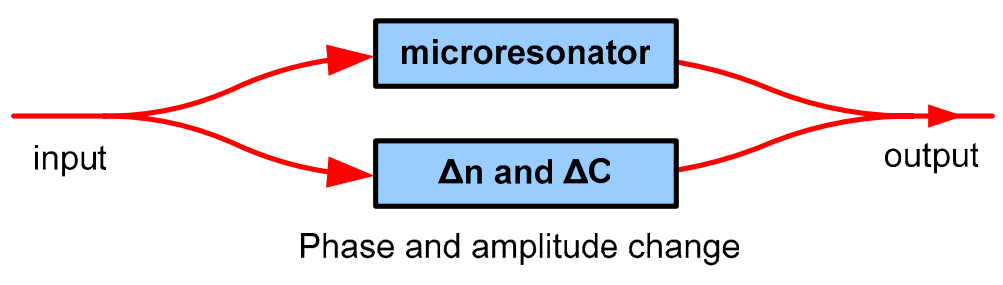

Phase and amplitude change

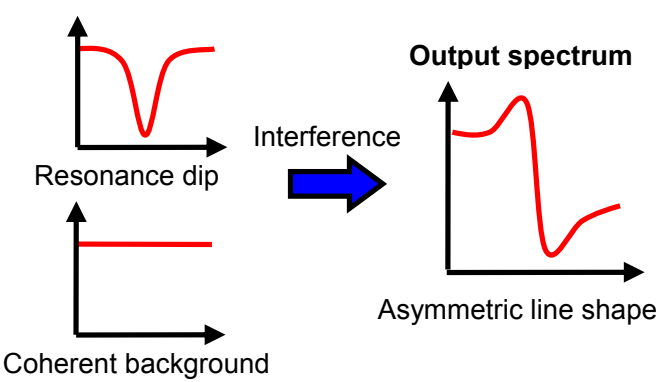

Coherent background

Figure 1: Illustration of Fano resonance asymmetric line shapes formation and tuning by using a Mach-Zehnder interferometer. One arm of the interferometer is coupled to a microresonator to generate a Lorentzian resonance field, whereas the other arm provides relative phase and amplitude control to the coherent background field.

\section{OPTICAL FANO RESONANCES CONCEPT AND MODELING}

We model optical Fano resonances in terms of light wave interference between an inverted Lorentzian resonance and a coherent background (Fig. 1). We express the interference between a unit-amplitude inverted Lorentzian resonance field $E_{r}$ and a coherent continuum background field $E_{c}$ as follows,

$$
\left|E_{r}+E_{c}\right|^{2}=\left|\left[\varepsilon / \sqrt{ }\left(\varepsilon^{2}+1\right)\right] \exp i \phi_{\mathrm{r}}+\mathrm{C} \exp \mathrm{i} \phi\right|^{2}
$$

where $\varepsilon=\left(\lambda-\lambda_{\mathrm{o}}\right) /(\Gamma / 2), \lambda_{\mathrm{o}}$ is the resonance wavelength in free space, $\Gamma$ is the resonance linewidth, $\phi_{\mathrm{r}}=\tan ^{-1} \varepsilon, \mathrm{C}$ is the relative amplitude of the background continuum, and $\phi$ is the relative phase of the continuum. We assume both $\mathrm{C}$ and $\phi$ are constants within a narrow spectral range. We expand the terms in (1) and obtain,

$$
\left|E_{r}+E_{c}\right|^{2}=(\mathrm{q}-\varepsilon)^{2} /\left(1+\varepsilon^{2}\right)+\left(\mathrm{C}^{2}-\varepsilon^{2}\right) \cos ^{2} \phi /\left(1+\varepsilon^{2}\right)+(\mathrm{C}+\cos \phi)^{2} \varepsilon^{2} /\left(1+\varepsilon^{2}\right)
$$

where $\mathrm{q}=\mathrm{C} \sin \phi$ is the Fano asymmetry parameter. The first term of (2) is the well-known Fano line shape, whereas the second and third terms represent a symmetric Lorentzian and a symmetric inverted Lorentzian.

Figure 2 shows the modeled asymmetric resonance lineshapes using (2), with $\lambda_{\mathrm{o}}=1558.42 \mathrm{~nm}, \mathrm{Q}=10^{4}, \Gamma=0.156 \mathrm{~nm}$, $\mathrm{C}=1$, for $\phi=0,0.5 \pi, \pi$, and $1.5 \pi$. Physically, the asymmetric resonance line shapes result from interference between the resonant field and the coherent continuum field with relative phase values $0<|\phi|<\pi$. Whereas, the symmetric Lorentzian dip $(\phi=0)$ results from a constructive interference between the inverted Lorentzian resonant field and the coherent continuum field. In contrast, the symmetric Lorentzian peak $(\phi=\pi)$ results from a destructive interference between the inverted Lorentzian resonant field and the coherent continuum field. It is worth mentioning that (2) results in characteristics distinguishable from those of standard Fano line shapes. At $\varepsilon=\mathrm{q},(2)$ results in finite intensities, yet Fano line shapes given by $(\mathrm{q}-\varepsilon)^{2} /\left(1+\varepsilon^{2}\right)$ alone gives a zero-intensity.

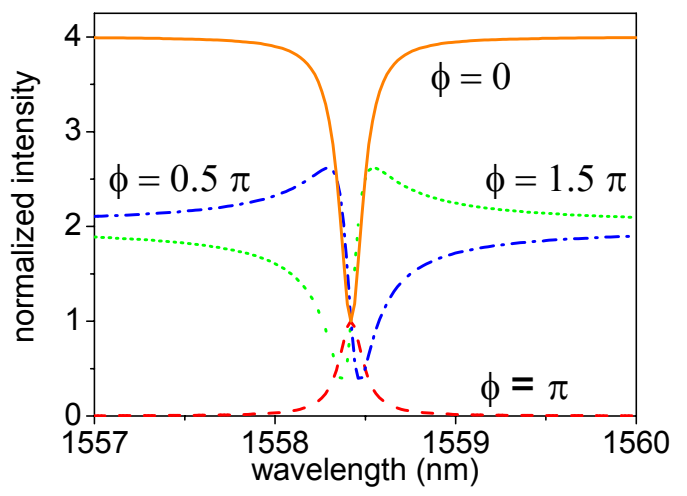

Figure 2: Modeled asymmetric resonance line shapes with $\phi=0$ (orange solid line), $0.5 \pi$ (blue dashed line), $\pi$ (red dashed line), and $1.5 \pi$ (green dashed line) . 


\section{FANO LINE SHAPES TUNING BY USING A MACH-ZEHNDER INTERFEROMETER}

Figure 3 illustrates Fano line shapes tuning by separately controlling $\phi$ and $\mathrm{C}$ using an MZI setup. Our interferometer comprises a resonance pathway given by a prism-coupled hexagonal micropillar resonator, and a coherent background pathway given by a phase shifter and a variable attenuator.

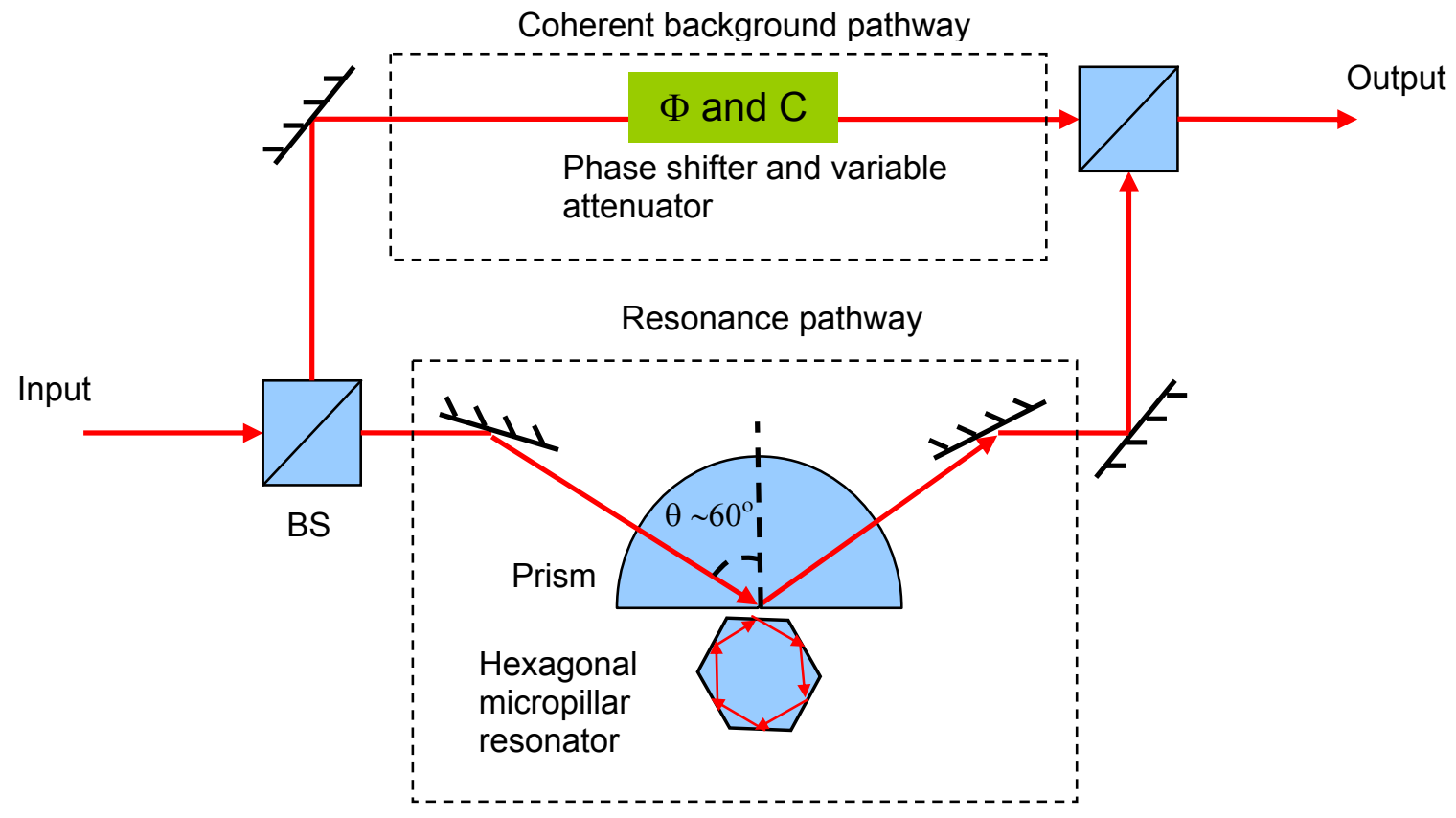

Figure 3: Illustration of the use of prism-coupled hexagonal micropillar resonator in the resonance pathway and Fano line shapes tuning by separately controlling $\phi$ and $\mathrm{C}$ in the coherent background pathway. BS: beam splitter

Comparing with conventional circular micropillar or microsphere resonators, polygonal micropillar resonators in the shape of square ${ }^{3,4}$, hexagonal ${ }^{8-10}$, and octagonal ${ }^{11}$, have the key merit of flat cavity sidewalls for the ease of evanescent coupling along relatively long lateral interaction lengths. Hence, hexagonal micropillar resonators offer alternative microresonators to microresonator-based applications. Previously ${ }^{9}$, we reported that hexagonal micropillar resonators exhibit resonances with 6-bounce ray orbits that are partially confined by total internal reflection at the six cavity sidewalls. Resonances occur only when the ray orbits are wavefront-matched with the input-coupled wavefronts upon each round trip. For a hexagonal micropillar resonator of a sidewall-to-sidewall distance $a$ and a refractive index $n$, the wavefront-matched 6-bounce round-trip optical path length is given $\operatorname{as}^{8,9} n \mathrm{~L}=n 3 a \sin \left(\theta+30^{\circ}\right)$, where $\theta$ is the ray incident angle.

Figure 4 shows a schematic of our MZI-microresonator experimental setup. A TM-polarized (electric field parallel to the micropillar axis) Gaussian beam from an external-cavity wavelength-tunable diode laser $(1510 \mathrm{~nm}-1580 \mathrm{~nm}$; linewidth $\sim 300 \mathrm{kHz})$ is weakly focused by an $\mathrm{f} / 81$ spherical lens with a cone angle of about $0.8^{\circ}\left(\approx 1 / \mathrm{e}^{2}\right.$ angular width). The laser beam is equally split by a 50/50 beam splitter. In one arm (the resonance pathway), the laser beam is focused onto a bulk fused-silica cylinder prism with an incident angle $\theta$ near $60^{\circ}$. Such $\theta$ favors the input coupling to 6-bounce ray orbits ${ }^{8,9}$. The illuminated prism is coupled to a commercially available fused-silica hexagonal optical fiber acting as a hexagonal micropillar resonator. The fiber sidewall and the prism flat surface are in contact near the prism origin. Inset (a) shows a top-view optical micrograph of the prism-coupled hexagonal fiber, with $a \approx 125 \mu \mathrm{m}$ (sidewall length $\approx$ $72 \mu \mathrm{m})$ and six rounded corners. In the other arm (the coherent background pathway), we employ two right-angled gold-coated glass prisms (with one of the prisms mounted on a piezo-electric transducer) to form a phase shifter. We also use a polarization analyzer as a variable optical attenuator. We then separately control the relative phase $\phi$ and the relative amplitude $\mathrm{C}$ between the MZI arms. The piezo-electric transducer has a resolution of $\sim 30 \mathrm{~nm}$, and thus the 
minimum delay increment is limited to $\sim 60 \mathrm{~nm}$ (corresponding to $\left.\Delta \phi \sim 2 \pi^{*}(60 \mathrm{~nm}) /(1550 \mathrm{~nm}) \sim 0.24 \mathrm{rad}\right)$. Light beams from the two arms are recombined by another $50 / 50$ beam splitter. The resultant interference fringe is sampled by a $62.5-\mu \mathrm{m}$ core multimode fiber after an analyzer. We set the two arms optical path lengths to be nearly balanced. The spectral resolution is about $0.02 \mathrm{~nm}$, which is only limited by the laser wavelength-tuning steps.

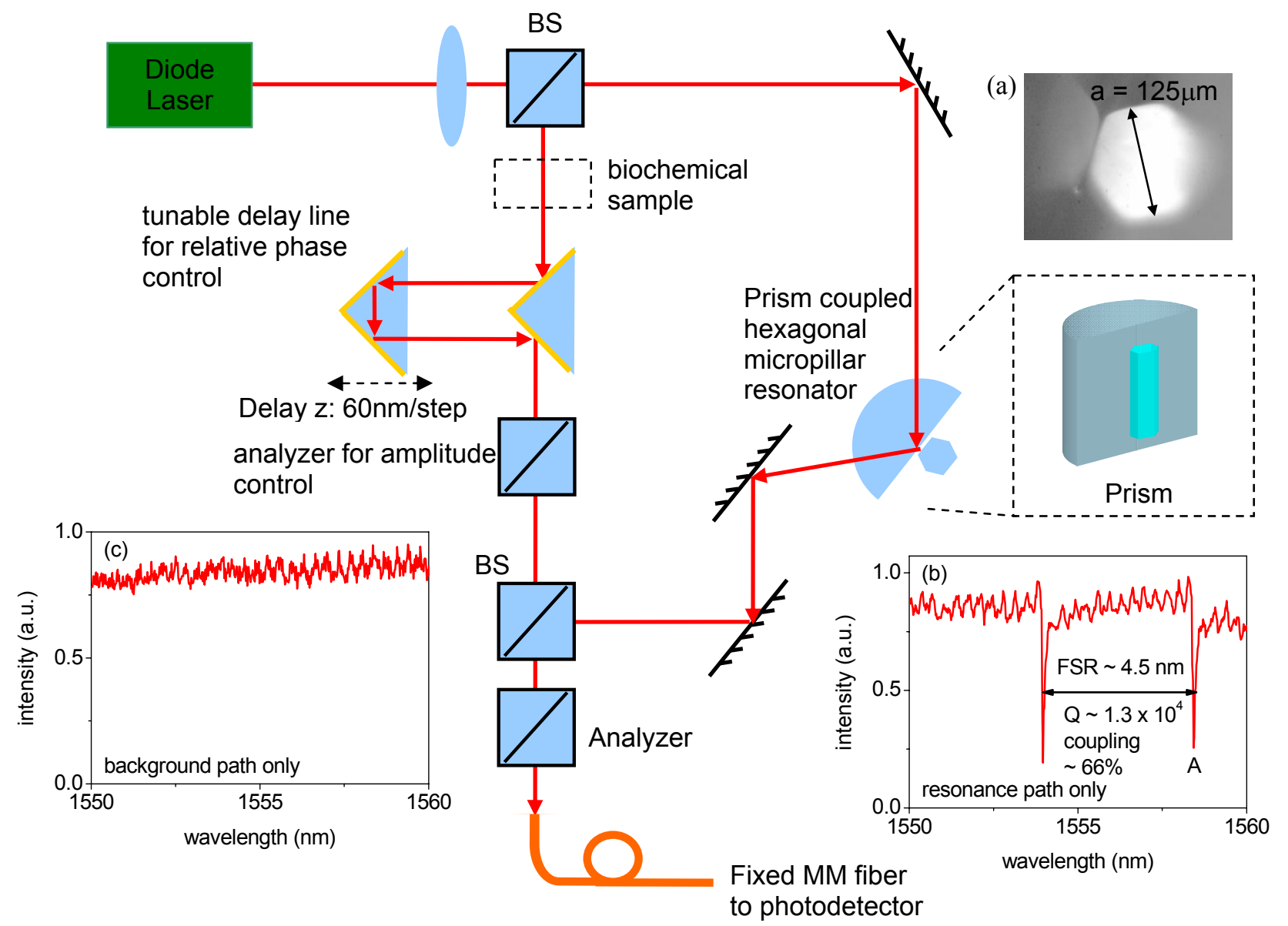

Figure 4: Schematic of the Mach-Zehnder interferometer-based Fano line shapes tuning experimental setup. Inset (a) shows a top-view optical micrograph of the prism-coupled hexagonal fiber. Insets (b) and (c) show typical TM-polarized output spectra of resonance-only and background-only . BS: beam splitter, MM fiber: multimode fiber (62.5 $\mu \mathrm{m}$ core diameter)

Insets (b) and (c) show typical measured TM-polarized interferometer output spectra obtained with the resonance path only and the background path only. The two spectra are set to approximately the same intensity (i.e. C $\sim 1$ ). The resonance-only spectrum reveals desirable singlemode resonances. The resonance line shapes are near symmetric inverted Lorentzian. The measured free spectral range (FSR) is about $4.5 \mathrm{~nm}$, consistent with the calculated FSR $\approx$ $\lambda^{2} /(n \mathrm{~L}) \approx 4.44 \mathrm{~nm}$ for a 6-bounce round-trip orbit with $\theta \sim 60^{\circ}$ and $n \sim 1.44$, where $\lambda(=1558.42 \mathrm{~nm})$ is the resonance wavelength in free space. Resonance A exhibits a $\mathrm{Q} \sim 1.3 \times 10^{4}$ and a coupling efficiency $\sim 66 \%$. The ripples in both spectra are due to residual etalon modulation within the laser diode.

Figures 5(a) - 5(e) show our initially measured interferometer output spectra with controllably tuned Fano line shapes at various relative delays. Here we set the coherent background intensity to be approximately twice the resonance path intensity. We tune the asymmetric line shapes from near symmetric dip to near symmetric peak and return to near symmetric dip. Fano asymmetric line shapes are discerned in Figs. 5(b) and 5(d). 


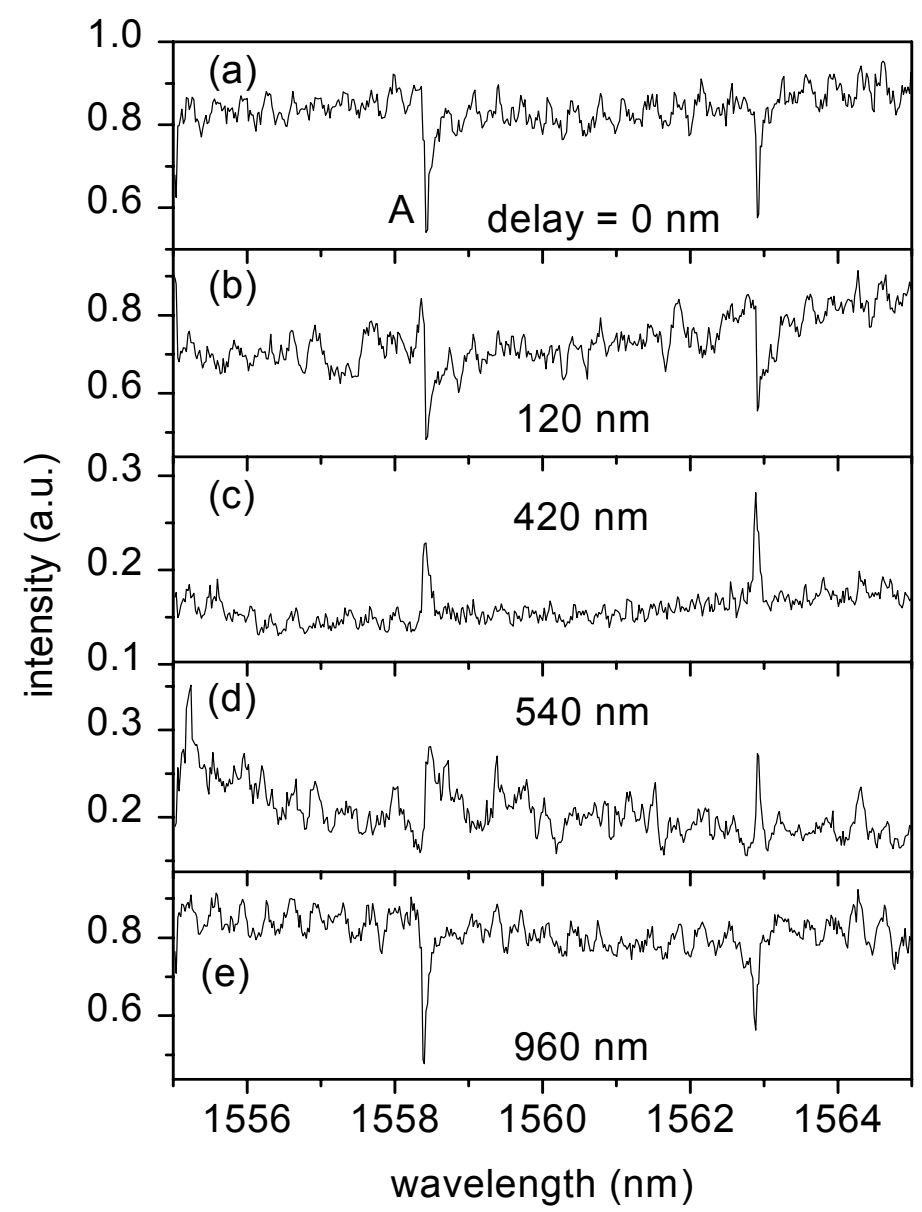

Figure 5: Initially measured resonance line shapes tuning. The coherent background intensity is approximately twice the resonance path intensity and the delay (a) $0 \mathrm{~nm}$, (b) $120 \mathrm{~nm}$, (c) $420 \mathrm{~nm}$, (d) $540 \mathrm{~nm}$, and (e) $960 \mathrm{~nm}$.

\section{POTENTIAL APPLICATIONS IN BIOCHEMICAL SENSING AND COMPARISON WITH RELATED TECHNIQUES}

Based on our initial experimental demonstration, we believe that by inserting a sample cell in the coherent background path (see Fig. 4), our resonance line shapes tuning technique should be applicable for sensing small phase shifts due to the presence of a certain biochemical content in the cell.

Conventionally, interferometry has long been employed for sensing small relative phase shifts. By inserting a (biochemical) sample into one arm of an MZI, the phase change can be represented as an intensity variation in the interferometer output. This conventional approach has an advantage that the sample can be readily inserted and removed from the interferometer arm. However, the free space interferometer arms alignment may be problematic and prone to mechanical instabilities.

Recently, high-Q microresonator-based sensing ${ }^{7}$ has attracted much research interest. When this method is applied for biochemical sensing, it imposes coating the entire microresonator with a thin-film of sample in order to induce an effective refractive index change that results in resonance wavelength shifts. The key merit is that an ultra high-Q microresonator (e.g. Q $\sim 10^{6}$ or beyond) offers an ultra-high sensitivity. However, the drawback is that the microresonator needs to be thoroughly cleaned or disposed after use. Furthermore, the sensor performance can also be limited by the laser wavelength instability, and hence a precision laser wavelength monitoring should be desirable. 
By contrast, we believe that our MZI-based microresonator technique offers essentially an interferometric method for sensing, yet with the potential benefit of using the asymmetric resonance line shapes change as an additional measure, which is less affected by laser wavelength instabilities.

Here we contrast more quantitatively our proposed technique for (biochemical) sensing with conventional interferometric method using MZI. We assume a refractive index shift $\Delta n=10^{-4}(n=1.33)$. We consider the sample has a thickness of $1 \mathrm{~mm}$, resulting in a phase shift $\Delta \phi$ of $\sim 0.4 \mathrm{rad}$ (at $1558.47 \mathrm{~nm}$ ). Following the measured spectra (Fig. 5), we assume a resonance wavelength at $1558.42 \mathrm{~nm}$ with a resonance $\mathrm{Q} \sim 10^{4}$. Using (2), we calculate the resonance line shapes with $\mathrm{C}=1$ and $\phi=\pi / 2$. Figure 6(a) shows the calculated asymmetric resonance line shape change under the phase shift of $0.4 \mathrm{rad}$. We note that the calculated intensity change at an off-resonance wavelength is essentially the same as conventional MZI response. We believe that the resonance line shape change is favorable for sensing applications.
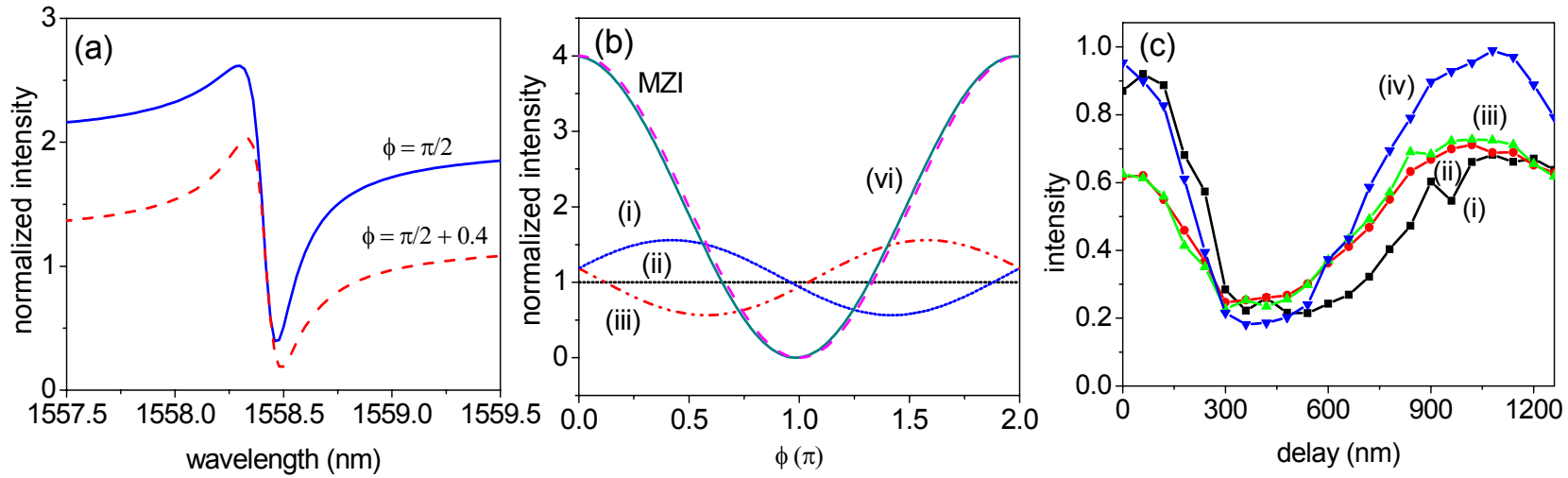

Figure 6: (a) Calculated Fano line shapes at $\phi=\pi / 2$ (blue solid line) and $\phi=\pi / 2+0.4$ (red dashed line). (b) Calculated intensity with relative phase at various probe wavelengths (i) $1558.45 \mathrm{~nm}$ (blue dotted line), (ii) $1558.47 \mathrm{~nm}$ (resonance wavelength, black dotted line), (iii) $1558.49 \mathrm{~nm}$ (red dashed line), and (iv) $1560.00 \mathrm{~nm}$ (off-resonance wavelength, green solid line). The MZI intensity response (purple dashed line) is shown for comparison. (c) Initially measured intensity as a function of delay at various probe wavelengths (i) $1558.38 \mathrm{~nm}$ (squares), (ii) $1558.40 \mathrm{~nm}$ (circles), (iii) $1558.42 \mathrm{~nm}$ (triangles), and (iv) $1560.42 \mathrm{~nm}$ (inverted triangles).

Figure 6(b) shows the calculated intensity against $\phi$ for our proposed technique at various wavelengths and for conventional MZI sensing. At an off-resonance wavelength, our technique matches that of conventional MZI-only method. However, with the probe wavelength approaches the resonance wavelength, our proposed method exhibits less sensitivity to relative phase shifts than conventional MZI. The phase response becomes flat at the resonance wavelength. Although our proposed approach only displays sensitivity as good as a conventional MZI, we believe that the flat response at a resonance wavelength offers a unique reference that is fixed in intensity under any degree of relative phase shifts. We could then have a MZI-based sensing technique that has a self-reference at the resonance wavelength.

Figure 6(c) shows our analysis of the initially measured spectra (Fig. 5) in terms of the measured intensities as a function of delay (phase shift) at various probe wavelengths near resonance A. However, unlike our modeling, the response curves measured near the resonance wavelength still display considerable modulations. Moreover, the response curves fail to display the expected $\pi$-phase shifted oscillations in our modeling (shown in Fig. 6(b)). Instead, all the extracted response curves essentially follow the same modulation. We attribute the modulation to perhaps a MZI-dominant effect results from possible alignment issues. Further experiments are needed to confirm our modeled Fano line shapes tuning and to substantiate the proposed technique as a useful MZI-microresonator based biosensing method.

\section{CONCLUSION}

We experimentally demonstrated controlled Fano line shapes tuning in prism-coupled silica hexagonal micropillar resonators using a Mach-Zehnder interferometer. Our experimental setup reveals singlemode resonances $\left(\mathrm{Q} \sim 1.3 \times 10^{4}\right)$ in hexagonal micropillar resonators that are input and output coupled near $60^{\circ}$. The sharp resonance line shapes 
observed in the interferometer output are characteristically asymmetric. By controlling the interfering coherent continuum field relative amplitude and relative phase, we demonstrated $2 \pi$ asymmetric resonance line shapes tuning. We believe that our technique of controlled resonance line shapes tuning deserves further investigation for potential applications in biochemical sensing as an alternative approach to conventional interferometric and microresonator-based methods.

\section{ACKNOWLEDGEMENTS}

This study was substantially supported by grants from the Research Grants Council of the Hong Kong Special Administrative Region, China (projects HKUST6166/02E \& 618505).

\section{REFERENCES}

1. U. Fano, "Effect of configuration interaction on intensities and phase shifts," Phys. Rev. 124, 1866 (1961).

2. S. Fan, "Sharp asymmetric line shapes in side-coupled waveguide-cavity systems", Appl. Phys. Lett. 80, 908 (2002).

3. H.T. Lee and A. W. Poon, "Fano resonances in prism-coupled square micropillars," Opt. Lett. 29, 5 (2004).

4. H.T. Lee, L. Zhou, and A. W. Poon, "Fano resonances in prism-coupled multimode square micropillar resonators," Opt. Lett. 30, 1527 (2005).

5. A. Chiba, H. Fujiwara, J.-I. Hotta, S. Takeuchi, and K. Sasaki, "Fano resonance in a multimode tapered fiber coupled with a microspherical cavity," Appl. Phys Lett. 86, 261106 (2005).

6. Y. Lu, J. Yao, X. Li, and P. Wang, "Tunable asymmetrical Fano resonance and bistability in a microcavity-resonator-coupled Mach-Zehnder interferometer," Opt. Lett. 30, 3069 (2005).

7. C. Y. Chao and L. J. Guo, "Biochemical sensors based on polymer microrings with sharp asymmetrical resonance," Appl. Phys. Lett. 83, 1527 (2003).

8. N. Ma, C. Li, and A. W. Poon, "Laterally coupled hexagonal micropillar resonator add-drop filters in silicon nitride," IEEE Photon. Technol. Lett. 16, 2487 (2004).

9. N. Ma, F. K. L. Tung, S. F. Lui, and A. W. Poon, "Hexagonal micro-pillar cavities: multimode resonances and open-loop resonance linewidth broadening," in Physics and Simulation of Optoelectronic Devices XI, Marek Osinski, Hiroshi Amano, Peter Blood ed., Proc. SPIE 4986, 153 (2003).

10. J. Wiersig, "Hexagonal dielectric resonators and microcrystal lasers," Phys, Rev. A. 67, 023807 (2003).

11. C. Li and A. W. Poon, "Experimental demonstration of waveguide-coupled round-cornered octagonal microresonators in silicon nitride," Opt. Lett. 30, 546 (2005). 